\title{
SURGICAL ANATOMY OF THE LEVATOR PALPEBRAE INSERTION*
}

\author{
BY \\ EDWARD EPSTEIN \\ Johannesburg, Union of South Africa
}

THE text-book description of the anatomy of the upper eyelid with particular reference to the septum orbitale (palpebral fascia) and especially the insertion of the levator muscle is unnecessarily vague or complicated from the surgical point of view.

Thus the septum orbitale is described by some authors as arising from the orbital margin and ending where it blends with the aponeurosis of the levator. Most, however, describe it as extending almost to the lower edge of the tarsal plate with which it blends. It has also been noted as having an attachment to the upper border of the tarsal plate.

The insertion of the levator is described as splitting into three laminae which have the following attachments:

(1) Skin.

(2a) Lower part of anterior surface of tarsal plate.

(2b) Osseous.

(3a) Upper margin of tarsal plate.

(3b) Upper palpebral conjunctiva and fornix.

Lamina (1) thus pierces the septum orbitale and then the orbicularis muscle to reach the skin. For Lamina (2) to reach the lower part of the tarsal plate, it must either pierce and lie anterior to the septum orbitale, or blend with it or pass down just posterior to it. These details are not well defined and it is impossible to dissect and separate these layers. That the levator aponeurosis should first pierce the palpebral fascia and then the orbicularis muscle is an unusual anatomical concept and difficult to comprehend. It is almost equivalent to saying that the recti muscles pierce the sclera to become attached to the limbus.

Dissections of fresh and cadaver material, however, have suggested the following simplification (Figs 1-6, overleaf):

The palpebral fascia extends as a continuous sheet, in some places thicker than others, from the periosteum of the orbital margin almost to the palpebral margins.

\footnotetext{
* Received for publication April 10, 1961.
} 


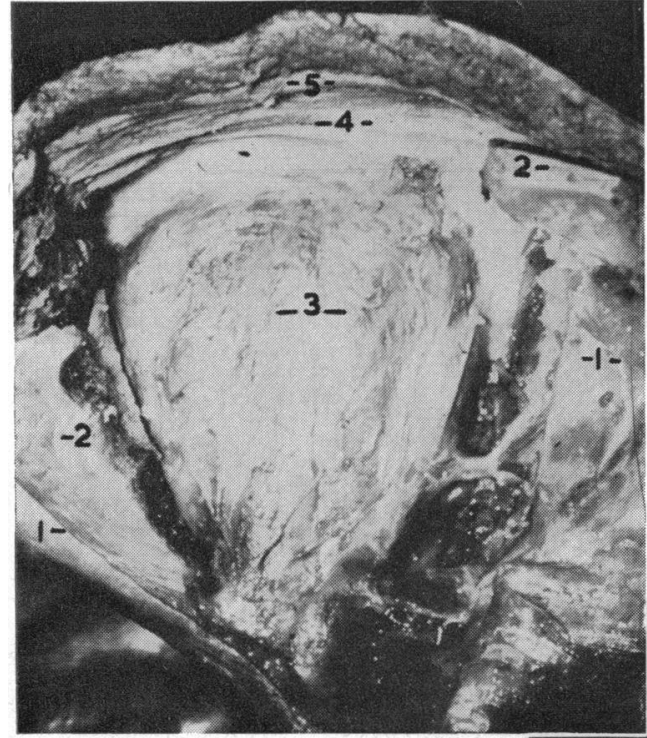

FIG. 1.-The dura ${ }^{1}$ and bone ${ }^{2}$ of the anterior fossa have been removed, exposing the periosteum of the roof of the orbit ${ }^{3}$, the orbital margin 4 , and the forehead 5 .

FIG. 2.-The periosteum of the orbital roof 3 has been almost completely excised, exposing the lacrimal gland 6 , orbital fat 7 , a small area of levator aponeurosis 8 , levator muscle ${ }^{9}$, and superior oblique 10 . A small strip of the palpebral fascia 11 is visible between the cut edge of the orbital periosteum and the levator aponeurosis.

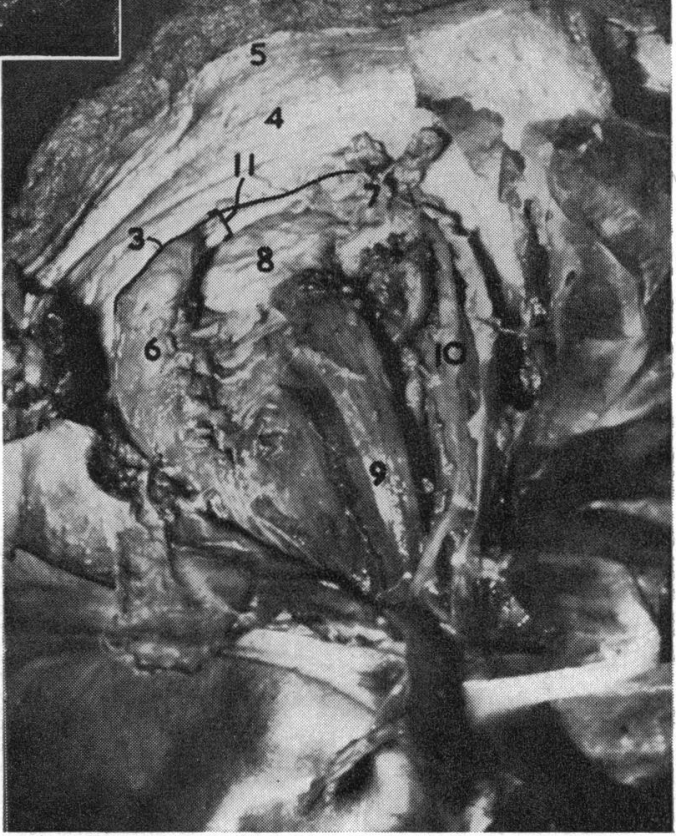

In the upper lid it blends with the lower quarter of the anterior surface of the tarsal plate. The pretarsal space therefore separates the upper three-quarters of this plate from this fascia. (In the lower lid the fascia blends completely with the anterior surface of the plate.)

The orbicularis oculi muscle lies anterior to the palpebral fascia. Fine septa extend from the fascia to the skin, dividing the orbicularis into arcuate bundles of muscle fibres. In some individuals and races (orientals) these septa are less developed than in others. The aponeurosis of the levator is inserted (without 


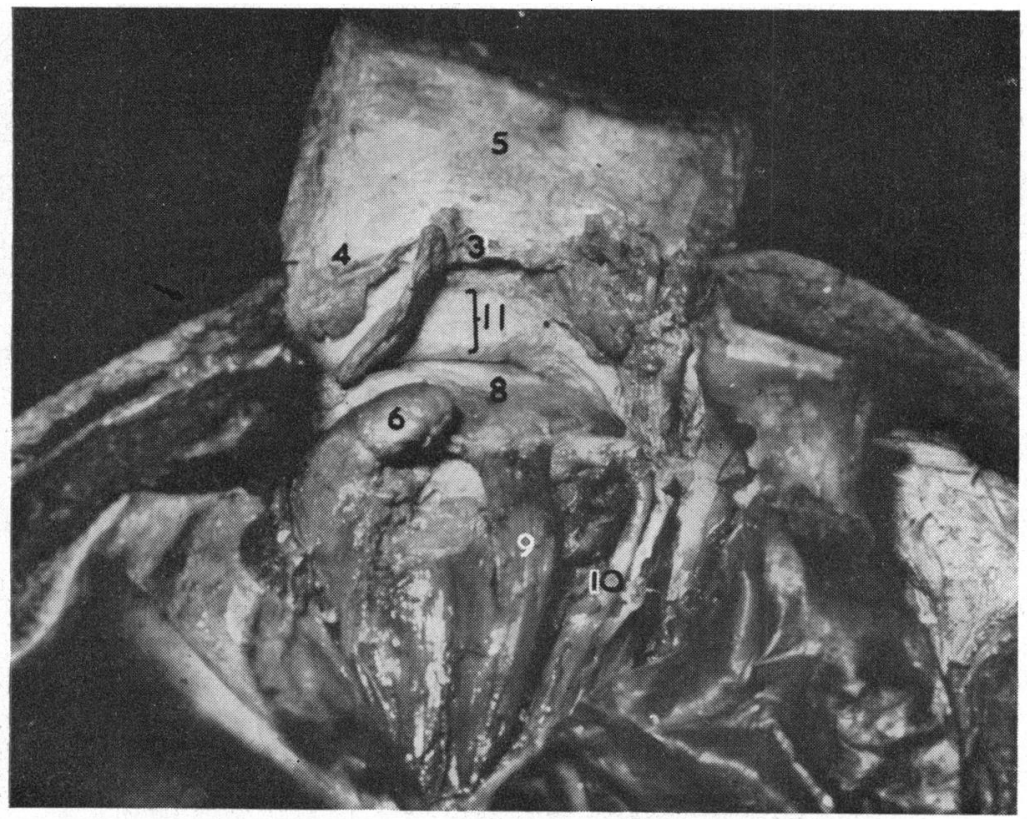

Fig. 4.-The levator has been divided, and the insertion of the aponeurosis into the palpebral fascia 11 is demonstrated.

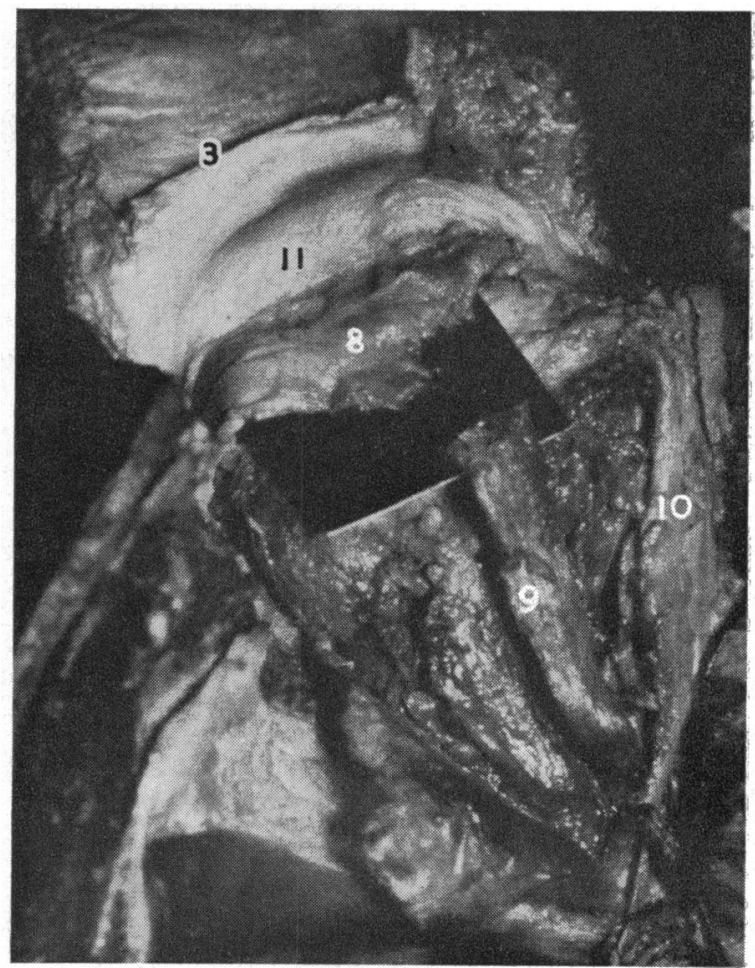

FIG. 3.-The tissues of the forehead have been incised vertically above the eye and turned downwards and forwards. The periosteum of the forehead 5 , the curve of the orbital margin 4 , and the cut free edge of the periosteum of the roof of the orbit 3 are now clearly defined. The palpebral fascia 11 (septum orbitale) is seen meeting the now fully-exposed upper surface of the levator aponeurosis 8 .

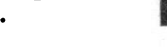




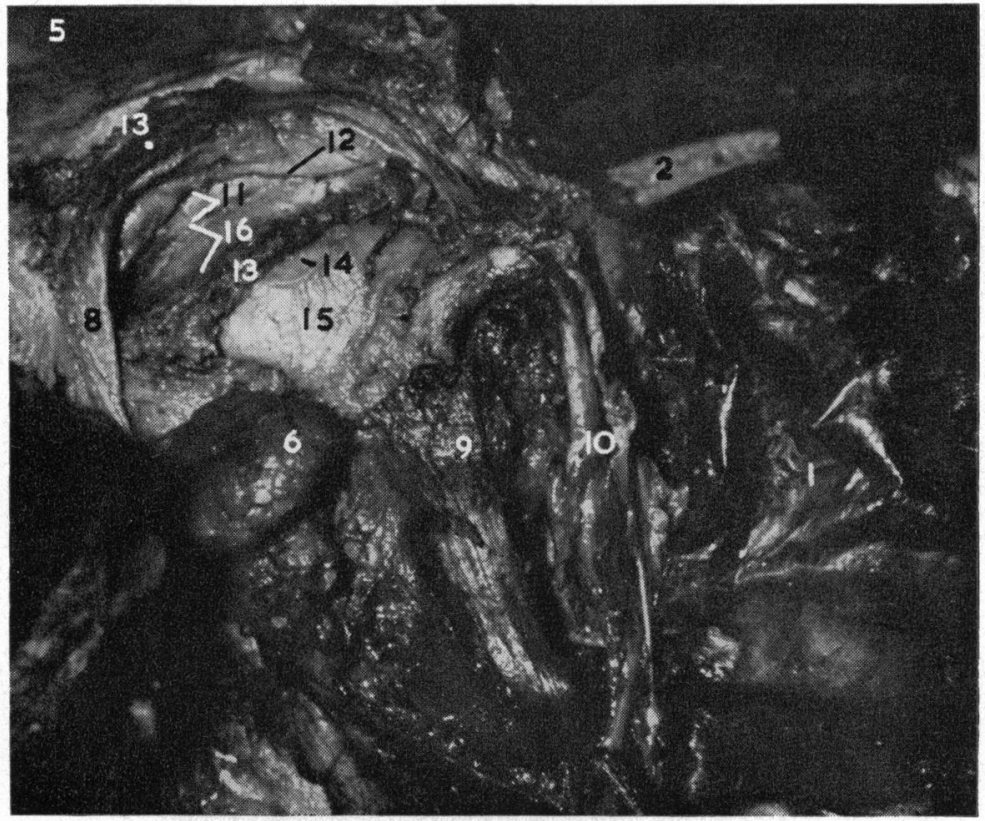

Fig. 5.-The aponeurosis 8 has been turned upwards and forwards to expose its undersurface, and most of the semilunar line 12 of its insertion into the palpebral fascia. The cut edges of the "cremaster tarsus" muscle13 can be seen on the everted aponeurosis and again just anterior to the upper fornix ${ }^{14}$ of the conjunctival sac, where the latter lies on the sclera 15 of the slightly distorted globe. The pretarsal space has been opened, showing the palpebral fascia 11 below the insertion of the aponeurosis 8 to the front, and the tarsal plate 16 with the "cremaster tarsus" inserted into its upper border behind.

splitting) into the palpebral fascia in a semilunar line midway between the bony orbital margin and the free edge of the lid.

I first became aware of this glistening semilunar line whilst doing the classical Blaskovicz operation for ptosis. Subsequent dissection of cadaver material showed that this line was the everted under-surface of the aponeurosis at its insertion.

The text-book's third lamina of the insertion of the levator is described as blending with a lamella of smooth muscle and inserting into the upper border of the tarsal plate. The ptosis of Horner's syndrome proves that any fibres from the aponeurosis attached to the upper tarsal border must be subordinate to the tone of the smooth muscle and hence not really necessary for the support of the tarsal plate; the smooth muscle lamina itself being sufficient. Likewise, if fibres from the aponeurosis really extended downwards with the septum orbitale to be attached to the lower anterior part of the tarsal plate, they would normally be relaxed and concertina'd to allow for a Horner's ptosis.

Histologically, the levator muscle is noted to terminate in the tendinous aponeurotic expansion, but the striated muscle fibres continue for a short distance 


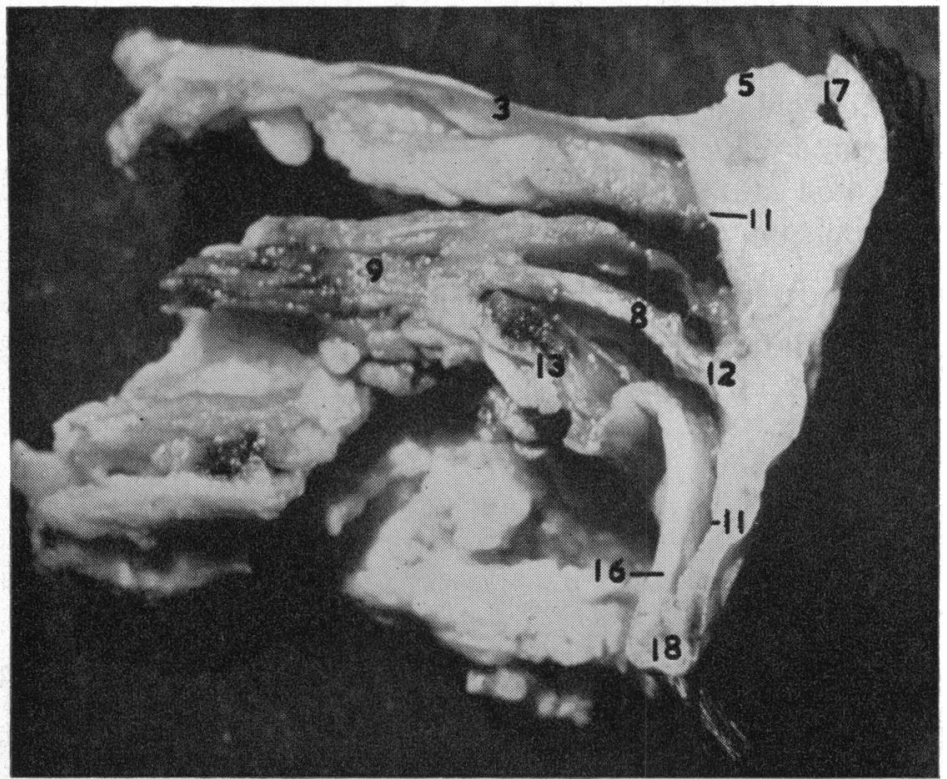

Fig. 6.-A vertical section through the brow ${ }^{17}$, upper lid (lash border ${ }^{18}$ ), and some upper orbital structures. The globe has been removed and the conjunctiva divided through the upper fornix. The tarsal plate 16 and pretarsal space are easily identifiable. The periosteum of the orbital margin ${ }^{4}$ and roof $^{3}$ can be seen, with the palpebral fascia ${ }^{11}$ arising from the former and passing downwards to the lid margin. The insertion 12 of the levator aponeurosis into this structure is demonstrated. The aponeurosis also has a well-developed though unusual fold on its upper surface, from the edge of which fine connective tissue extends towards the orbital fat and palpebral fascia. The bursa-like space on the upper surface of the aponeurosis is demonstrated. In the process of fixation the tissue has been distorted a little. The lid sulcus and brow have been flattened out so that the septum orbitale has been angled backwards.

beneath the aponeurosis and then give place to a layer of smooth muscle fibreMüller's superior palpebral muscle. This layer passing downwards and forwards to its insertion, curves over and just deep to the superior fornix and palpebral conjunctiva to which it is blended by areolar tissue. This is, therefore, a separate muscle with a separate nerve supply, and a more descriptive name would be the "cremaster tarsus" muscle. The action of the levator muscle is thus to pull on the palpebral fascia and fold it upon itself. Through the medium of the "cremaster tarsus" muscle the pull is also transmitted to the tarsal plate. The attachments of the palpebral fascia to the canthal ligaments act as check ligaments. The general attachments of the skin to the underlying orbicularis muscle and the septa from the palpebral fascia account for the superior lid sulcus. The tone of the "cremaster tarsus" accounts for the fine secondary sulci in the skin just below the main lid sulcus.

The practical significance of this description is that, in the surgery of the levator for the correction of ptosis, the aponeurosis must be detached from the palpebral fascia at the semilunar line, resected, and re-sutured to this line. Müller's muscle (the cremaster tarsus) must be resected proportion- 
ately, otherwise a Horner-like ptosis remains. The length of the lid (palpebral fascia) is undisturbed so that closure of the lid and lid movements on looking up and down are natural, provided the levator itself has reasonable power, tone, and elasticity. The lid margin is not distorted. The lid sulcus is created automatically without any complicated through-andthrough suturing.

To enter the correct plane for dissection the procedure is as follows:

The lid is everted over a Desmarres retractor. The conjunctiva is incised at the upper border of the tarsal plate and peeled off from the underlying "cremaster tarsus" towards the fornix (Fig. 7). The tarsal plate is then incised just within the upper edge with the plate pulled forwards, so that as the incision is deepened it enters the pretarsal space without damaging the palpebral fascia. It is then simple to strip the smooth muscle layer from the palpebral fascia (Fig. 8) until the white glistening under-surface of the aponeurosis presents itself at the semilunar line (Fig. 9).

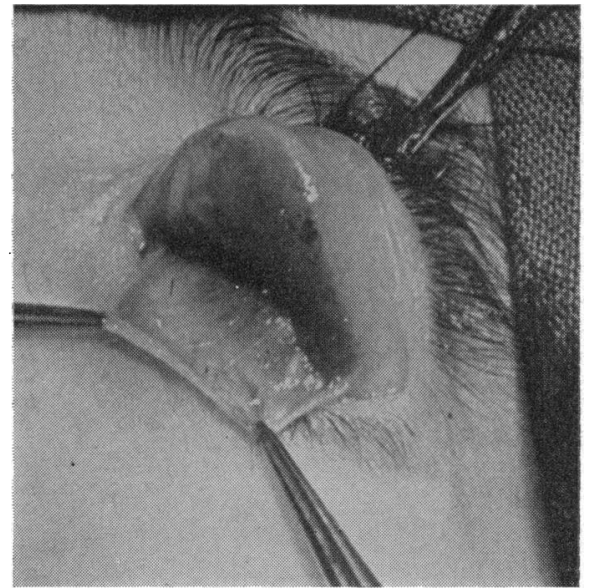

Fig. 7.-The upper lid is everted over a Desmarres retractor and the conjunctival layer has been detached from the upper tarsal border and reflected. The cornea is faintly visible through the conjunctiva.

FIG. 8.-Müller's muscle ("cremaster tarsus") has been reflected. The lower part of the palpebral fascia is exposed and a little of the under-surface of the levator aponeurosis is visible.

FIG. 9.-The "cremaster tarsus" is now fully exposed and its origin from the white shiny under surface of the levator aponeurosis is indicated by the forceps. The insertion of the aponeurosis into the palpebral fascia is in shadow and the semilunar line is just visible.
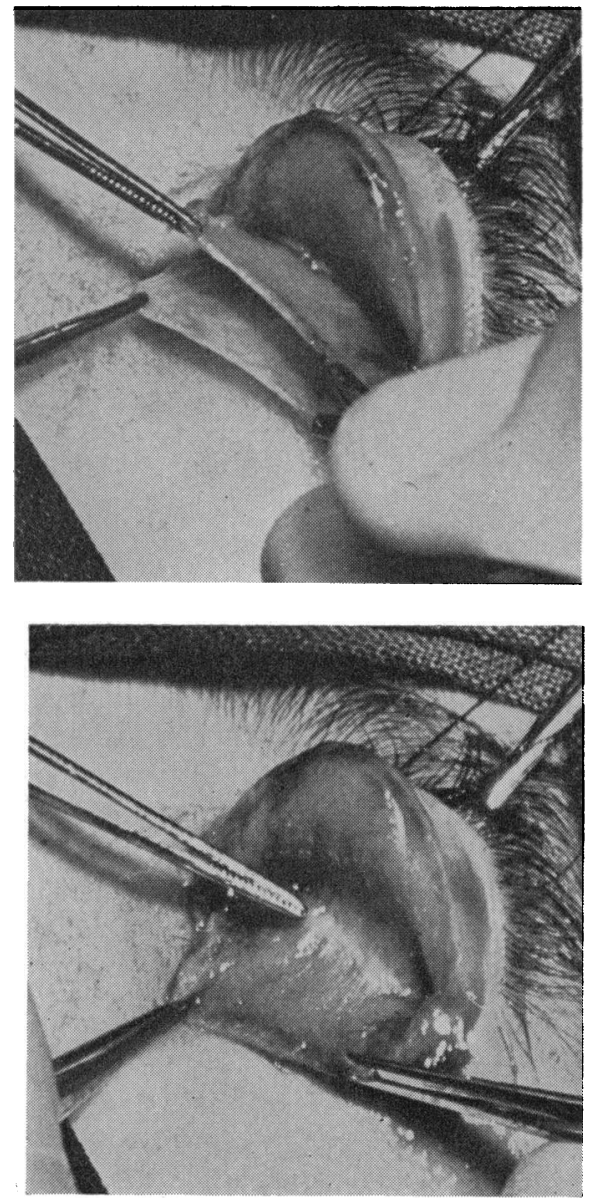
The aponeurosis can be picked up with sutures or fine forceps and incised (Fig. 10). It is then easy to introduce a muscle hook and complete with scissors. The upper surface of the aponeurosis has an appearance as if a bursa had been opened (Fig. 11). The orbital fat lies above contained in a thin envelope of connective tissue and does not prolapse if this layer is not damaged.

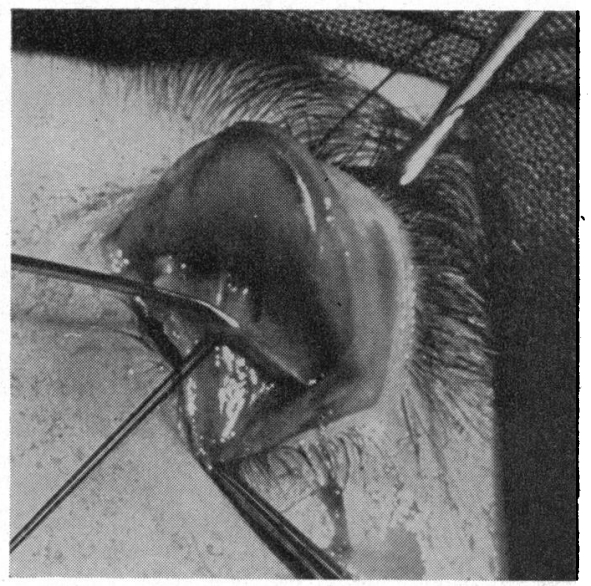

FIG. 10.-A stitch has been placed in the aponeurosis, which has been drawn down and divided at the semilunar line. A probe lies through this opening.

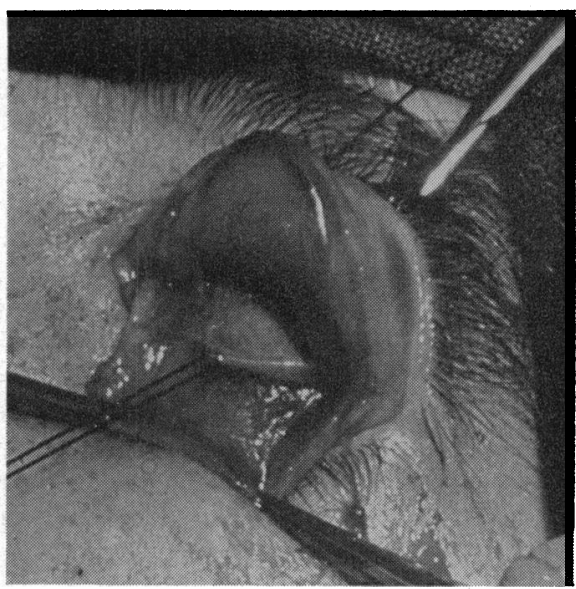

FIG. 11.-The detachment of the aponeurosis from the palpebral fascia has been enlarged, and the upper bursa-like surface of the aponeurosis is visible.

\section{Summary}

The levator palpebrae superioris muscle ends in an aponeurosis that is inserted into the palpebral fascia in a semilunar line midway between the orbital margin and the palpebral margin. The name "cremaster tarsus" muscle is suggested as being more descriptive of Müller's superior palpebral muscle. It arises from the terminal striated fibres beneath the aponeurosis and is inserted into the upper tarsal border.

The palpebral fascia is described as a layer of connective tissue extending from the orbital margin to the palpebral margin. The term "septum orbitale" must either be considered synonymous with the term "palpebral fascia", or clearly defined as limited to the thicker upper part of that membrane between the orbital margin and the insertion of the levator aponeurosis.

I am grateful to Prof. P. V. Tobias for allowing me to do this work in the Anatomy Department of the Witwatersrand Medical School, to Mr. R. Klomfas for technical assistance, to Mr. S. Dry for the histological preparations, and to the Medical Photographic Department and Mr. Shevitz in particular for the illustrations. 